EMERSON OLIVERA ${ }^{1}$

AdRIANA CheBAR LOZINSKY ${ }^{2}$

Claudia Cristina Palos ${ }^{3}$

Daniela D'Amelo Melara Ribero ${ }^{4}$

Angela Mara Bentes de Souza ${ }^{5}$

Caio Parente Barbosa ${ }^{6}$

\title{
Influência do índice de massa corporal na incontinência urinária feminina
}

\author{
Body mass index influence in female urinary incontinence
}

Artigo original

Palavras-chave

Incontinência urinária de esforço Índice de massa corporal

Obesidade

Severidade Qualidade de vida

Keywords

Stress urinary incontinence Body mass index

Obesity

Severity

Quality of life

\section{Resumo}

OBJETIVOS: avaliar e comparar os efeitos do índice de massa corporal (IMC) sobre severidade da incontinência urinária (IU) feminina por meio do questionário de qualidade de vida King's Health Questionnaire (KHQ), variáveis do estudo urodinâmico e dados da anamnese. MÉTODOS: estudo clínico transversal. Foram selecionados 65 pacientes com incontinência urinária de esforço (IUE) que foram divididas em três grupos: Grupo I (IMC entre 18 e $25 \mathrm{~kg} / \mathrm{m}^{2}$ ); Grupo II (IMC entre 25 e $\left.30 \mathrm{~kg} / \mathrm{m}^{2}\right)$ e Grupo III (IMC>30 kg/m²). Os domínios do KHQ foram comparados entre esses grupos. Além disso, alguns dados da anamnese e do estudo urodinâmico (presença de noctúria, enurese, urgência e urge-incontinência) foram também relacionados ao IMC calculando-se o OR (Odds Ratio). O IMC, na presença e na ausência de contrações não inibidas do detrusor, bem como no VLPP (valsalva leak point pressure) $<60$ ou $\geq 60$ $\mathrm{cmH}_{2} \bigcirc$ foi avaliado. Por fim, foram realizados testes de correlação do IMC com os nove domínios do KHQ a fim de se evidenciar alguma associação. RESULTADOS: o KHQ foi incapaz de registrar, em qualquer um de seus domínios, deterioração da qualidade de vida das mulheres com IU na medida em que ocorreu elevação do IMC. Encontramos OR para a presença de enurese em relação ao IMC de 1,003 [IC: 0,897-1,121], valor $p=0,962$. Para a noctúria, - OR foi de 1,049 (IC: 0,933-1, 18), valor $p=0,425$. O valor de OR=0,975 (IC: 0,826-1, 15 1), valor $p=0,762$ foi encontrado para a urgência. No que se refere à urge-incontinência, encontrou-se $O R=0,978$ (IC: 0,85-1, 126), valor $\mathrm{p}=0$,76. Estudou-se o IMC nos grupos com e sem contrações não-inibidas do músculo detrusor e foram encontradas, respectivamente, medianas de $26,4 \pm 4,8$ e $28,3 \pm 5,7 \mathrm{~kg} / \mathrm{m}^{2}(\mathrm{p}=0,6)$. De forma semelhante, as medianas do IMC nos grupos com $V L P P<60$ e $\geq 60 \mathrm{cmH}_{2} \bigcirc$ foram, respectivamente, de $29,6 \pm 4,1$ e $27,7 \pm 5,7 \mathrm{~kg} / \mathrm{m}^{2}(\mathrm{p}=0,2)$. Finalmente, não tivemos êxito em demonstrar associação do IMC com qualquer um dos nove domínios do KHQ por meio da correlação de Spearman. CONCLUSÃO: não houve associação dos escores do KHQ com o IMC. Também não houve correlação entre os parâmetros clínicos da anamnese e do estudo urodinâmico com o IMC.

\section{Abstract}

PURPOSE: to evaluate and compare the effects of body mass index (BMII) on the severity of female urinary incontinence (UI) using the quality of life questionnaire King's Health Questionnaire (KHQ), variables of urodynamic studies and the medical history taken. METHODS: cross-sectional clinical study. We selected 65 patients with stress urinary incontinence (SUI) who were divided into three groups: Group I (BMI: 18-25 kg/m²), Group II (BMI: 25-30 kg/m²) and Group III $\left(\mathrm{BMl}>30 \mathrm{~kg} / \mathrm{m}^{2}\right)$. The $\mathrm{KHQ}$ domains were compared between these groups. In addition, some clinical history urodynamic data (presence of nocturia, enuresis, urgency and urge incontinence) were also related to BMI by calculating the Odds Ratio (OR). The BMI in the presence and absence of non-inhibited detrusor contractions and Valsalva leak point pressure $(V L P P)<60$ or $\geq 60 \mathrm{cmH}_{2} \mathrm{O}$ were evaluated. Finally, the correlation between $\mathrm{BMI}$ and the nine $\mathrm{KHQ}$ domains has been tested in order to detect some association. RESULTS: the KHQ did not record deterioration of quality of life in women with $\mathrm{UI}$ with increasing BMI in any of its areas. The OR for the presence of enuresis in relation to a BMI was 1.003 [Cl: 0.897-1.121], $p=0.962$. The OR for nocturia was 1.049 [Cl: 0.933-1.18], $p=.425$. The OR for urgency was $0.975[\mathrm{Cl}: 0.826-1.151], p=0.762$, and the $\mathrm{OR}$ for incontinence was $0.978[\mathrm{Cl}: 0.85-1.126], p=0.76$. We studied
Rua das Figueiras, 1.800, Bloco A, apto. 94 - Campestre CEP 09080-301 - Santo André (SP), Brasil E-mail: emerson_oliveira@terra.com.br Os autores asseguram que, neste estudo, não há situação que configure conflito de interesses

Recebido

$17 / 8 / 09$

Aceito com modificacões

$28 / 9 / 10$
Disciplina de Ginecologia Patológica e Reprodução Humana do Setor de Uroginecologia e Cirurgia Vaginal da Faculdade de Medicina do ABC - FMABC - Santo André (SP), Brasil.

Professor Colaborador; Doutor do Departamento de Obstetrícia e Ginecologia da Faculdade de Medicina do ABC - FMABC - Santo André (SP), Brasil; Chefe do Setor de Uroginecologia e Cirurgia Vaginal da Faculdade de Medicina do ABC - FMABC - Santo André (SP), Brasil.

${ }^{2}$ Médica Residente do Departamento de Pediatria da Universidade Federal de São Paulo - UNIFESP - São Paulo (SP). Brasil.

${ }^{3}$ Médica Residente do Departamento de Obstetrícia e Ginecologia da Faculdade de Medicina do ABC - FMABC - Santo André (SP), Brasil.

${ }^{4}$ Médica Residente do Departamento de Obstetrícia e Ginecologia da Faculdade de Medicina do ABC - FMABC - Santo André (SP),

Brasil.

5 Professora Adjunto da Disciplina de Ginecologia Patológica e Reprodução Humana da Faculdade de Medicina do ABC - FMABC - Santo André (SP), Brasil.

- Professor Regente da Disciplina de Ginecologia Patológica e Reprodução Humana da Faculdade de Medicina do ABC - FMABC Santo André (SP), Brasil. 
the BMI in patients with and without non-inhibited detrusor contractions and detected medians of $26.4 \pm 4.8$ and $28.3 \pm 5.7 \mathrm{~kg} / \mathrm{m}^{2}, \mathrm{respectively}(\mathrm{p}=0.6)$. Similarly, the median BMI values for the groups with VLPP $<60$ and $\geq 60 \mathrm{cmH}_{2} \bigcirc$ were $29.6 \pm 4.1$ and $27.7 \pm 5.7 \mathrm{~kg} / \mathrm{m}^{2}$, respectively $(\mathrm{p}=0.2)$. Finally, we failed to demonstrate an association between BMI and any of the nine KHQ domains by means of the Spearman correlation. CONCLUSION: there was no association of $\mathrm{KHQ}$ scores with BMI. There was also no correlation between the parameters of clinical history and of the urodynamic study with BMI.

\section{Introdução}

Para a mulher, além do desconforto higiênico, a incontinência urinária (IU) determina problemas sociais, sexuais, psíquicos e econômicos ${ }^{1}$. A obesidade é considerada fator de risco significante e independente para a IU em mulheres ${ }^{2-8}$. Comparando-a com os outros fatores de risco (idade avançada, raça branca, paridade e número de partos vaginais) ${ }^{9}$, a obesidade é a que está mais relacionada à perda urinária ${ }^{4}$. Estima-se que $46 \mathrm{a}$ $67 \%$ dessas mulheres têm IU ${ }^{10}$. A obesidade é problema comum de saúde e, atualmente, tem alta prevalência em todo o mundo (mais de $50 \%$ na última década) ${ }^{11-}$ ${ }^{14}$. Em 2002, estimativas da Organização Mundial da Saúde (OMS) indicavam a existência de mais de 1 bilhão de adultos portadores dessa morbidade no globo. Estimativas recentes do governo americano apontam que, nos Estados Unidos, mais de $25 \%$ das mulheres são obesas, sendo que, a cada ano, aproximadamente 2,6 milhões de pessoas morrem em consequência do excesso de peso ${ }^{11}$.

O índice de massa corporal (IMC) é considerado método objetivo e acurado para avaliar o peso de cada indivíduo. É calculado dividindo-se o peso em quilogramas pela altura em metros ao quadrado ${ }^{15}$. Estudo recente demonstrou estreita relação entre o aumento do IMC e a presença de IU, assim como o aparecimento de sintomas urinários como urgência miccional, aumento da frequência, noctúria e tenesmo vesical ${ }^{16}$. No entanto, os trabalhos que buscam relacionar a severidade da IU com o IMC apontam que a questão ainda é passível de discussão. Nesse contexto, um recente estudo que incluiu 2.875 mulheres apontou que a obesidade é fator associado à severidade da incontinência urinária de esforço (IUE) ${ }^{17}$. Corroborando com os resultados do estudo anterior, outros autores também tiveram êxito em demonstrar essa associação ${ }^{18-22}$. Por outro lado, alguns estudos não encontraram relação entre a severidade dos sintomas urinários e a obesidade ${ }^{23,24}$. Face ao exposto, interessou-nos neste estudo avaliar as possíveis associações entre o IMC e a IU feminina.

\section{Métodos}

Foram selecionadas 65 mulheres com diagnóstico de IU atendidas entre Janeiro e Julho de 2008 no Ambulatório de Uroginecologia e Cirurgia Vaginal do
Hospital Estadual Mario Covas, instituição vinculada à Faculdade de Medicina do ABC (FMABC). Como critérios de inclusão foram estabelecidos: diagnóstico urodinâmico de IU e a capacidade de ler, compreender e responder a um questionário. As que concordaram em participar do estudo assinaram termo de consentimento livre e esclarecido previamente aprovado pelo Comitê de Ética em Pesquisa da FMABC sob o número 033-2008.

As pacientes foram divididas em três grupos: Grupo I (pacientes com IMC entre 18 e $25 \mathrm{~kg} / \mathrm{m}^{2}$ ); Grupo II (pacientes com IMC entre 25 e $30 \mathrm{~kg} / \mathrm{m}^{2}$ ) e Grupo III (pacientes com IMC $>30 \mathrm{~kg} / \mathrm{m}^{2}$ ). A homogeneidade entre os grupos foi assegurada no que se referia à idade, paridade, tipo de parto, estadiamento do prolapso genital e presença de cirurgias prévias para a IU (Tabela 1$)$. As médias de idades das pacientes foram $51,06 \pm 11 ; 54,6 \pm 8,9 ; 53,6 \pm 8,5$ anos, respectivamente, e a mediana da paridade foi de três partos para cada um dos grupos. Havia 41 pacientes na pós-menopausa e 24 na menacme. O IMC médio foi de $28,3 \pm 4,5 \mathrm{~kg} / \mathrm{m}^{2}$.

Após a anamnese, o exame físico geral (incluindo a medida do IMC) e o exame ginecológico (procedimentos da rotina do atendimento ambulatorial dessas pacientes) aplicou-se às voluntárias o questionário King's Health Questionnaire (KHQ), específico para a IU e já validado para a língua portuguesa ${ }^{25}$. O KHQ é composto por 30 perguntas arranjadas em nove domínios. Relatam, respectivamente, a percepção da saúde, o impacto da incontinência, as limitações do desempenho das tarefas, a limitação física, a limitação social, o relacionamento pessoal, as emoções, o sono e a energia e as medidas de gravidade. Existe também uma escala de sintomas, composta pelos seguintes itens: frequência urinária,

Tabela 1 - Características clínicas dos grupos estudados

\begin{tabular}{|c|c|c|c|c|}
\hline Variável & GI & GII & GIII & Valor p \\
\hline Média da idade (anos)* & 51,06 & 54,61 & 53,60 & 0,6 \\
\hline Paridade (mediana)* & 3 & 3 & 3 & 0,8 \\
\hline Partos vaginais (mediana)* & 2 & 2 & 2 & 0,5 \\
\hline Partos fórcipes (mediana)* & 0 & 0 & 0 & 0,9 \\
\hline Cesarianas (mediana)* & 0 & 0 & 0 & 0,4 \\
\hline Cirurgias prévias** & 4 & 4 & 5 & 0,8 \\
\hline $\begin{array}{l}\text { Estadiamento do prolapso } \\
\text { genital }{ }^{* *}\end{array}$ & $\begin{array}{c}0 / 1-6 \\
\| 1-9 \\
\text { III/IV-2 }\end{array}$ & $\begin{array}{c}0 / 1-11 \\
\|-10 \\
\| I I / V-1\end{array}$ & $\begin{array}{c}0 / 1-9 \\
\| 1-13 \\
\text { III/IV-3 }\end{array}$ & 0,8 \\
\hline
\end{tabular}

* Valor $\mathrm{p}$ obtido pelo teste de Kruskall-Wallis; ${ }^{* *}$ valor $\mathrm{p}$ obtido pelo teste do $\chi^{2}$. 
noctúria, urgência, hiper-reflexia vesical, IUE, enurese noturna, incontinência no intercurso sexual, infecções urinárias e dor na bexiga. Há, também, um espaço para a paciente relatar qualquer outro problema que possa ter relacionado com a bexiga ${ }^{25}$. A todas as respostas são atribuídos valores numéricos, somados e avaliados por domínio. Os valores são, então, calculados por meio de fórmula matemática, obtendo-se, assim, o escore de qualidade de vida, que varia de 0 a 100 , considerando-se que quanto maior o número obtido, pior a qualidade de vida ${ }^{25}$.

Todas as pacientes também foram submetidas ao estudo urodinâmico. O valor da pressão de perda (VLPP) e a presença de contrações não inibidas do músculo detrusor (CNI) foram registrados. Adicionalmente, alguns dados da anamnese, como a presença de noctúria, enurese, urgência e urge-incontinência, foram também correlacionados ao IMC. Por fim, realizamos testes de correlação do IMC com os nove domínios do KHQ a fim de se evidenciar alguma associação.

A análise estatística foi realizada com os softwares Statistical Package for the Social Sciences (SPSS) versão 15.0 e GraphPad Prism 4.0. As variáveis quantitativas foram comparadas utilizando-se o teste de Kruskal-Wallis. Para as variáveis enurese, noctúria, urgência miccional e urge-incontinência foi realizado o cálculo da OR a partir da aplicação do modelo de regressão logística binária. Avaliamos a mediana do IMC nos grupos com e sem CNI,

Tabela 2 - Odds ratio para as variáveis estudadas de acordo com índice de massa corporal

\begin{tabular}{lccc}
\hline Variáveis & Odds ratio & $\begin{array}{c}\text { Infervalo de } \\
\text { confiança }\end{array}$ & Valor p* \\
\hline Enurese & 1,0 & $0,89-1,12$ & 0,9 \\
Noctúria & 1,04 & $0,93-1,18$ & 0,4 \\
Urgência & 0,97 & $0,82-1,15$ & 0,7 \\
Urge- & 0,97 & $0,85-1,12$ & 0,7 \\
\hline incontinência & & & \\
\hline
\end{tabular}

* Valor p: calculado a partir de regressão logística binária. bem como naqueles com VLPP $<60$ e $\geq 60 \mathrm{cmH}_{2} \mathrm{O}$ pela aplicação do teste não paramétrico de Mann-Whitney. Finalmente, para as análises de correlação utilizamos o teste não paramétrico de Spearman. O nível de rejeição da hipótese de nulidade foi fixado em $5 \%$.

\section{Resultados}

Estudou-se o IMC nos grupos com e sem CNI e encontramos, respectivamente, medianas de 26,4 44,8 e $28,3 \pm 5,7 \mathrm{~kg} / \mathrm{m}^{2}$ (Mann Whitney $U=435$; valor de $\mathrm{p}=0,6)$. De forma semelhante, as medianas do IMC nos grupos com VLPP $<60$ e $\geq 60 \mathrm{cmH}_{2} \mathrm{O}$ foram, res-

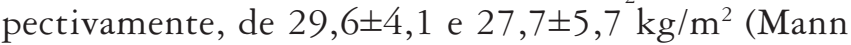
Whitney $U=327,5$; valor de $p=0,2)$. Por meio do cálculo da OR pode-se perceber que não houve associação entre a presença de enurese, noctúria, urgência e urge-incontinência com o IMC (Tabela 2).

O questionário KHQ foi aplicado e, na Tabela 3, observa-se a variação dos domínios conforme os valores do IMC. Nessas análises, pode-se perceber que o KHQ foi incapaz de registrar, em qualquer um de seus domínios, deterioração da qualidade de vida das mulheres com IU na medida em que ocorreu elevação do IMC.

Finalmente, buscamos correlacionar o IMC aos nove domínios do KHQ utilizando a correlação de Spearman. Entretanto, a exemplo das análises anteriores, não obtivemos êxito em demonstrar qualquer associação. Os resultados obtidos foram: saúde geral $(\rho($ rho $)=0,2$; valor $\mathrm{p}=0,08)$; impacto da incontinência ( $\rho$ (rho) $=-0,04$; valor $\mathrm{p}=0,7)$; limitação de atividades diárias $(\rho($ rho $)=-$ $0,01$; valor $\mathrm{p}=0,9)$; limitação física $(\rho(\mathrm{rho})=-0,04$; valor $\mathrm{p}=0,6$ ); limitação social ( $\rho$ (rho) $=-0,15$; valor $p=0,2)$; relacionamentos pessoais ( $\rho($ rho $)=-0,14$; valor $\mathrm{p}=0,2)$; emoções $(\rho$ (rho $)=-0,14$; valor $\mathrm{p}=0,2)$; sono e disposição $(\rho($ rho $)=-0,09$; valor $p=0,4)$ e medidas de severidade $(\rho($ rho $)=0,017$; valor $\mathrm{p}=0,8)$.

Tabela 3 - Medianas dos domínios do King's Health Questionnaire (KHQ) de acordo com o IMC (kg/m²)

\begin{tabular}{|c|c|c|c|c|}
\hline \multirow{2}{*}{ Domínios } & \multicolumn{3}{|c|}{ IMC } & \multirow{2}{*}{ Valor $\mathrm{p}^{*}$} \\
\hline & 18-25 & $25-30$ & $>30$ & \\
\hline Saúde geral & $50 \pm 20,8$ & $50 \pm 30,1$ & $50 \pm 23,8$ & 0,1 \\
\hline Impacto da incontinência & $100 \pm 31,0$ & $100 \pm 22,2$ & $100 \pm 31,5$ & 0,8 \\
\hline Limitação de atividades diárias & $66,7 \pm 27,9$ & $66,7 \pm 34,4$ & $66,7 \pm 38,9$ & 0,4 \\
\hline Limitação física & $83,3 \pm 28,3$ & $66,6 \pm 35,8$ & $66,6 \pm 33,1$ & 0,5 \\
\hline Limitação social & $33,3 \pm 31,4$ & $22,2 \pm 27,4$ & $22,2 \pm 20,7$ & 0,2 \\
\hline Relacionamentos pessoais & $66,6 \pm 42,6$ & $16,6 \pm 38,3$ & $33,3 \pm 33,7$ & 0,2 \\
\hline Emoções & $77,8 \pm 36,0$ & $66,6 \pm 34,1$ & $55,6 \pm 38,7$ & 0,4 \\
\hline Sono e disposição & $33,3 \pm 41,7$ & $66,6 \pm 45,1$ & $66,6 \pm 45,1$ & 0,3 \\
\hline Medidas de severidade & $66,6 \pm 28,4$ & $75 \pm 25,7$ & $66,6 \pm 22,6$ & 0,5 \\
\hline
\end{tabular}

IMC: índice de massa corporal; ${ }^{*}$ Teste de Kruskal-Wallis. 


\section{Discussão}

Este estudo buscou estabelecer uma relação entre a severidade da IU avaliada por parâmetros urodinâmicos (número de $\mathrm{CNI}$ e $\mathrm{VLPP}<60 \mathrm{cmH}_{2} \mathrm{O}$ ) e clínicos (anamnese e KHQ) e o IMC. Entretanto, por meio desses parâmetros, fomos incapazes de demonstrar qualquer relação.

Acredita-se que o excesso de peso aumente a pressão abdominal durante as atividades diárias, o que promoveria elevação na pressão vesical e maior mobilidade da uretra e colo vesical e, consequentemente, a $\mathrm{IU}^{26}$. Portanto, é bastante plausível a hipótese de que, em pacientes com sobrepeso e obesidade, a severidade da incontinência seja maior.

Alguns trabalhos na literatura apontam uma relação direta entre o grau de obesidade e a severidade da $\mathrm{IU}^{17-}$ 22. Em um ensaio clínico randomizado que incluiu 650 pacientes com IUE, a severidade da doença foi mensurada pelo número de episódios de perda involuntária de urina durante o dia. Os pesquisadores encontraram que a severidade da IUE foi positivamente associada com o IMC $(p=0,0003)^{22}$. No entanto, outros estudos mostram dados conflitantes. Por meio da análise de 1.155 questionários respondidos por mulheres que foram estratificadas por idade (25-39, 40-54, 55-69 e 70-84 anos) e pelo IMC (obesidade, obesidade severa e obesidade mórbida) não se observou qualquer relação entre a severidade dos sintomas urinários e o grau de obesidade após a aplicação do modelo de regressão logística. Digno de nota é o achado do aumento da prevalência de IUE em mulheres obesas mórbidas e obesas severas quando comparadas às obesas ${ }^{23}$. Ao encontro do estudo anterior, 268 mulheres foram avaliadas em desenho transversal do tipo caso-controle e também não houve êxito em se demonstrar qualquer associação da severidade dos sintomas urinários com o grau de obesidade ${ }^{24}$.

Nos últimos anos, com o advento da cirurgia bariátrica para a obesidade mórbida, muitos trabalhos têm sido direcionados no sentido de se avaliar os efeitos da perda de peso em mulheres portadoras de $\mathrm{IU}^{27-30}$, o que, em nosso entender, representa o desenho ideal para a resposta dos questionamentos envolvidos em nosso artigo. Um estudo envolvendo 12 mulheres com IU (IMC no pré-operatório de $\left.49,4 \pm 7,9 \mathrm{~kg} / \mathrm{m}^{2}\right)$ que se submeteram à cirurgia bariátrica demonstrou diminuição dos sintomas urinários em 9 das 12 mulheres $(\mathrm{p}=0,004)$. Sete de dez pacientes com IUE e oito de nove pacientes com urge-incontinência tiveram a completa resolução dos sintomas ${ }^{27}$. Em um coorte de 138 mulheres obesas mórbidas que perderam $50 \%$ ou mais de seu peso após cirurgia bariátrica, observou-se que a prevalência dos sintomas foi significativamente reduzida: de $61,1 \%$ durante o período pré-operatório para 11,6\% no pósoperatório $(\mathrm{p}=0,001)^{28}$. Em outro trabalho, demonstrou-se, após a cirurgia, redução da prevalência da IU e da severidade da doença avaliadas pela aplicação de questionários de qualidade de vida específicos para a IU. Entretanto, neste estudo, a redução dos escores não foi correlacionada com a magnitude da mudança no IMC ${ }^{29}$.

Os efeitos de uma dieta de baixa caloria e de exercícios físicos foram avaliados em 64 mulheres incontinentes, e observou-se que a diminuição de pelo menos $5 \%$ do IMC associou-se com a redução nos valores registrados no pad test (diferença média de $19 \mathrm{~g} ; \mathrm{p}<0,001$ ) e melhora nas medidas de qualidade de vida ${ }^{30}$.

Finalmente, nossos resultados permitem-nos concluir que não houve associação dos escores do KHQ com o IMC, assim como não houve correlação entre os parâmetros clínicos da anamnese e do estudo urodinâmico com o IMC.

\section{Referências}

1. Ternent L, Vale L, Buckley B, Glazener C. Measuring outcomes of importance to women with stress urinary incontinence. BJOG. 2009; 1 16(5):719-25

2. Must A, Spadano J, Coakley EH, Field AE, Colditz G, Dietz WH. The disease burden associated with overweight and obesity. JAMA. 1999;282(16):1523-9.

3. Brown JS, Grady D, Ouslander JG, Herzog AR, Varner RE, Posner SF. Prevalence of urinary incontinence and associated risk factors in postmenopausal women. Heart \& Estrogen/Progestin Replacement Study (HERS) Research Group. Obstet Gynecol. 1999;94(1):66-70.

4. Brown JS, Seeley DG, Fong J, Black DM, Ensrud KE, Grady D. Urinary incontinence in older women: who is at risk? Study of Osteoporotic Fractures Research Group. Obstet Gynecol. 1996;87/5 Pt 1):715-21.
5. Diokno AC, Brock BM, Herzog AR, Bromberg J. Medical correlates of urinary incontinence in the elderly. Urology. 1990;36(2): 129-38.

6. Milsom I, Ekelund P, Molander U, Arvidsson L, Areskoug B. The influence of age, parity, oral contraception, hysterectomy and menopause on the prevalence of urinary incontinence in women. J Urol. 1993; 149(6):1459-62.

7. Wingate $L$, Wingate $M B$, Hassanein $R$. The relation between overweight and urinary incontinence in postmenopausal women: a case control study. Menopause 1994;|(4): 199-203.

8. Mommsen S, Foldspang A. Body mass index and adult female urinary incontinence. World J Urol. 1994;12(6):319-22.

9. Melville JL, Katon W, Delaney K, Newton K. Urinary incontinence in US women: a population-based study. Arch Intern Med. $2005 ; 165(5): 537-42$. 
10. Gerten KA, Richter HE, Burgio KL, Wheeler TL, Goode PS, Redden DT. Impact of urinary incontinence in morbidly obese women versus women seeking urogynecologic care. Urology. 2007;70(6): 1082-5.

11. Costa PR, Assis AM, Silva Mda C, Santana ML, Dias JC, Pinheiro $S M$, et al. Change in anthropometric parameters: the impact of a nutritional intervention program and physical exercise on adult women. Cad Saude Publica. 2009;25(8): 1763-73

12. Matsushita $Y$, Mizoue $T$, Takahashi $Y$, Isogawa $A$, Kato $M$, Inove $M$, et al. Taste preferences and body weight change in Japanese adults: the JPHC Study. Int J Obes (Lond). 2009;33(10):1191-7.

13. Pigeot I, Barba G, Chadjigeorgiou C, de Henauw S, Kourides $Y$, Lissner $L$, et al. Prevalence and determinants of childhood overweight and obesity in European countries: pooled analysis of the existing surveys within the IDEFICS Consortium. Int J Obes (Lond). 2009;33(10): 1 103-10.

14. Hernández-Mijares A, Solá-Izquierdo E, Ballester-Mechó F, MaríHerrero MT, Gilabert-Molés JV, Gimeno-Clemente N, et al. Obesity and overweight prevalences in rural and urban populations in East Spain and its association with undiagnosed hypertension and Diabetes Mellitus: a cross-sectional population-based survey. BMC Res Notes. 2009;2:151.

15. WHO Expert Consultation. Appropriate body-mass index for Asian populations and its implications for policy and intervention strategies. Lancet. 2004;363(9403):157-63.

16. Moller LM, Lose $G$, Jorgensen T. Risk factors of lower urinary tract symptoms in women aged $40-60$ years. Ugeskr Laeger. 2001; 163(47):6598-601.

17. Minassian VA, Stewart WF, Wood GC. Urinary incontinence in women: variation in prevalence estimates and risk factors. Obstet Gynecol. 2008; 111 (2 Pt 1):324-31.

18. Nygaard IE, Heit M. Stress urinary incontinence. Obstet Gynecol. 2004; 104(3):607-20.

19. Mishra GD, Hardy R, Cardozo L, Kuh D. Body weight through adult life and risk of urinary incontinence in middle-aged women: results from a British prospective cohort. Int J Obes (Lond). 2008;32(9): 1415-22
20. Hannestad YS, Rortveit G, Daltveit AK, Hunskaar S. Are smoking and other lifestyle factors associated with female urinary incontinence? The Norwegian EPINCONT Study. BJOG. 2003;1 10(3):247-54.

21. Burgio KL, Zyczynski H, Locher JL, Richter HE, Redden DT, Wright $\mathrm{KC}$. Urinary incontinence in the 12-month postpartum period. Obstet Gynecol. 2003;102(6):1291-8.

22. Richter HE, Burgio KL, Brubaker L, Moalli PA, Markland AD, Mallet $V$, et al. Factors associated with incontinence frequency in a surgical cohort of stress incontinent women. Am J Obstet Gynecol. 2005; 193(6):2088-93.

23. Whitcomb EL, Lukacz ES, Lawrence JM, Nager CW, Luber KM. Prevalence and degree of bother from pelvic floor disorders in obese women. Int Urogynecol J Pelvic Floor Dysfunct. 2009;20(3): 289-94.

24. Ku JH, Shin JW, Oh SJ, Kim SW, Paick JS. Clinical and urodynamic features according to subjective symptom severity in female urinary incontinence. Neurourol Urodyn. 2006;25(3):215-20.

25. Fonseca ESM, Camargo ALM, Castro RA, Sartori MGF, Fonseca MCM, Lima GR, et al. Validação do questionário de qualidade de vida (King's Health Questionnaire) em mulheres brasileiras com incontinência urinária. Rev Bras Ginecol Obstet. 2005;27(5):235-42.

26. Alling Moller L, Lose $G$, Jorgensen T. Risk factors for lower urinary tract symptoms in women 40 to 60 years of age. Obstet Gynecol. 2000;96(3):446-51

27. Bump RC, Sugerman HJ, Fantl JA, McClish DK. Obesity and lower urinary tract function in women: effect of surgically induced weight loss. Am J Obstet Gynecol. 1992;167(2):392-7.

28. Deitel M, Stone E, Kassam HA, Wilk EJ, Sutherland DJ. Gynecologicobstetric changes after loss of massive excess weight following bariatric surgery. J Am Coll Nutr. 1988;7(2):147-53.

29. Burgio KL, Richter HE, Clements RH, Redden DT, Goode PS. Changes in urinary and fecal incontinence symptoms with weight loss surgery in morbidly obese women. Obstet Gynecol. 2007; $110(5): 1034-40$.

30. Auwad W, Steggles P, Bombieri L, Waterfield M, Wilkin T, Freeman R. Moderate weight loss in obese women with urinary incontinence: a prospective longitudinal study. Int Urogynecol J Pelvic Floor Dysfunct. 2008;19(9):1251-9. 